\title{
Servo design and analysis for the Thirty Meter Telescope primary mirror actuators
}

\author{
Peter M. Thompson ${ }^{\mathrm{a}}$, Douglas G. MacMynowski ${ }^{\mathrm{b}}$, Martin W. Regehr ${ }^{\mathrm{c}}$, \\ M. Mark Colavita ${ }^{\mathrm{c}}$, and Mark J. Sirota ${ }^{\mathrm{d}}$ \\ ${ }^{a}$ Systems Technology, Inc., 13766 S. Hawthorne Blvd., Hawthorne, CA 90250 \\ ${ }^{\mathrm{b}}$ California Institute of Tech., Dept. of Control and Dyn. Systems, Pasadena, CA 91125 \\ ${ }^{c} J e t$ Propulsion Laboratory, 4800 Oak Grove Dr., Pasadena, CA, USA 91109 \\ ${ }^{\mathrm{d}}$ Thirty Meter Telescope Corp., Pasadena, CA, USA
}

\begin{abstract}
The Thirty Meter Telescope has 492 primary mirror segments, each incorporated into a Primary Segment Assembly (PSA), each of which in turn has three actuators that control piston, tip, and tilt, for a total of 1476 actuators. Each actuator has a servo loop that controls small motions (nanometers) and large motions (millimeters). Candidate actuators were designed and tested that fall into the categories of "hard" and "soft," depending on the offload spring stiffness relative to the PSA structural stiffness. Dynamics models for each type of actuator are presented, which respectively use piezo-electric transducers and voice coils. Servo design and analysis are presented that include assessments of stability, performance, robustness, and control structure interaction. The analysis is presented for a single PSA on a rigid base, and then using Zernike approximations the analysis is repeated for 492 mirror segments on a flexible mirror cell. Servo requirements include low-frequency stiffness, needed for wind rejection; reduced control structure interaction, specified by a bound on the sensitivity function; and mid-frequency damping, needed to reduce vibration transmission. The last of these requirements, vibration reduction, was found to be an important distinguishing characteristic for actuator selection. Hard actuators have little inherent damping, which is improved using PZT shunt circuits and force feedback, but still these improvements were found to result in less damping than is provided by the soft actuator. Results of the servo analysis were used for an actuator down-select study.
\end{abstract}

Keywords: Extremely large telescopes, Primary mirror control system, Dynamic models, Control systems

\section{INTRODUCTION}

The Thirty Meter Telescope (TMT) is a segmented primary mirror telescope currently being designed for construction on Mauna Kea in Hawaii. ${ }^{[1]}$ The primary mirror will consist of 492 hexagonal segments. An image of the mirror cell and the elevation axis structure is in Figure 1a. This image is from the ANSYS finite element model developed at TMT.

Each mirror segment is supported on a Segment Support Assembly, which together with a mirror segment forms a Primary Segment Assembly (PSA), illustrated in Figure 1b. This image is from the NASTRAN finite element model developed at HYTEC, Inc. ${ }^{[2]}$ Example frequency responses for the mirror cell and PSA are also included in Figure 1. The frequency responses are discussed later in the text. Three actuators on each PSA control tip, tilt, and piston degrees of freedom. The remaining three degrees of freedom, clocking and translation in the plane of the segment, are not actively controlled.

There are 3 nested levels of control that maintain the shape and position of the segments forming the primary mirror; the total system is called the M1 Control System (M1CS): 1) The slowest acting is the warping harnesses on each mirror segment, which use information from the Alignment and Phasing System to correct the local figure of the individual segment, and is updated perhaps once every two weeks. 2) The global loop controller maintains the overall shape of the M1 mirror system based on 2272 edge sensors, correcting for slow deformations due to gravity and thermal changes, and faster deformations due to wind-induced pressure distributions. The bandwidth of the global loop controller is designed to be about $1 \mathrm{~Hz}$. The outputs of the global loop controller are position commands for the 1476 PSA actuators. 3) The servo loop controller is a position command system that acts locally about each actuator. The position sensor is an encoder with a resolution of $\sim 1 \mathrm{~nm}$. The servo actuators have two stages: a precision actuator designed to meet an RMS tracking error of $4.5 \mathrm{~nm}$, and an offload mechanism designed to meet a travel range of $5 \mathrm{~mm}$.

Ground-based and Airborne Telescopes III, edited by Larry M. Stepp, Roberto Gilmozzi, Helen J. Hall Proc. of SPIE Vol. 7733, 77332F · C 2010 SPIE · CCC code: 0277-786X/10/\$18 · doi: 10.1117/12.857371 


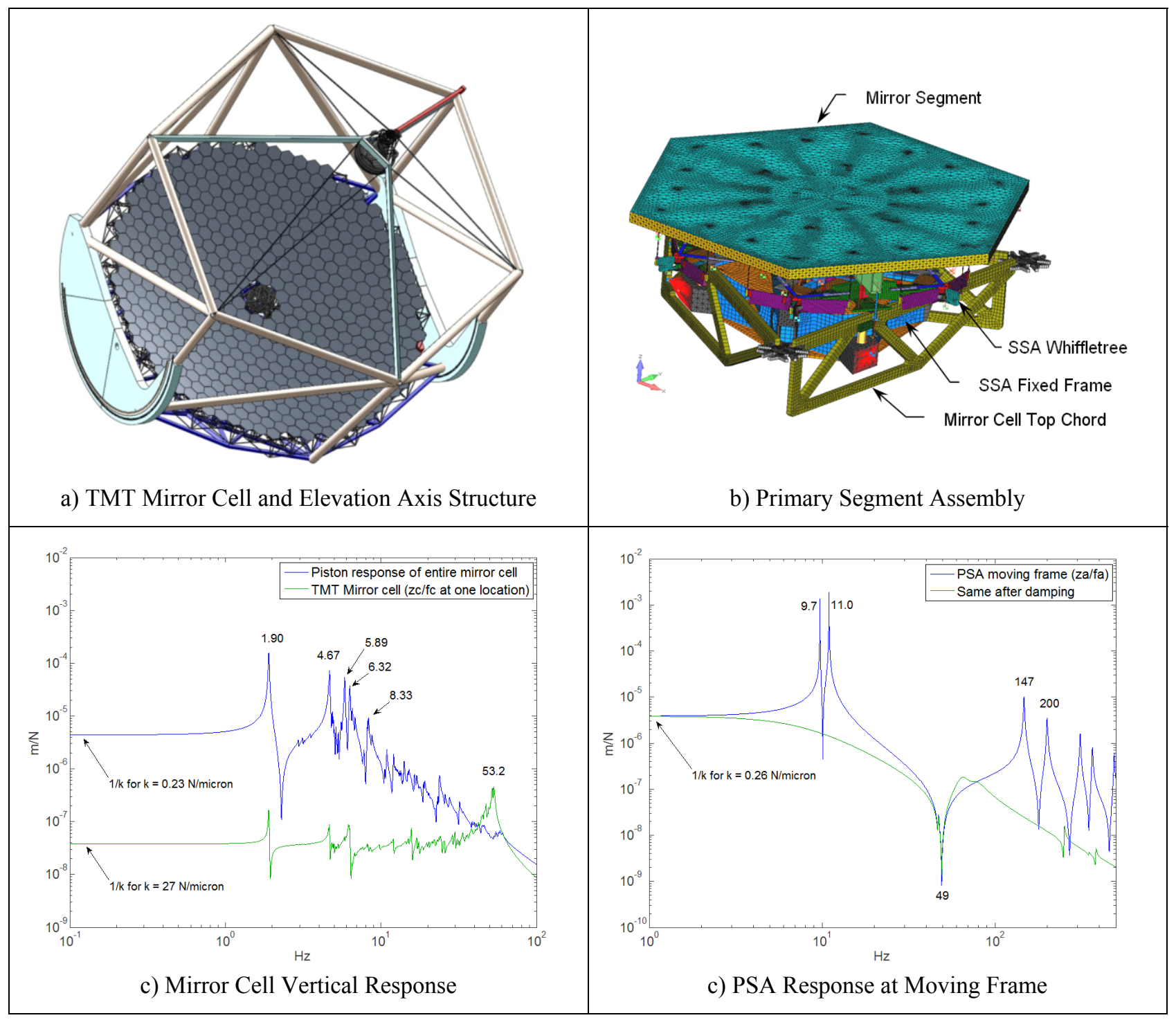

Figure 1: Primary Segment Assembly and TMT Mirror Cell

It is the servo loop controller - the innermost loop - that is the subject of this paper. Three candidate designs have been under consideration for the PSA actuators. The three names are defined below:

(VC) voice coil for precision control with stepper motor offload (soft actuator)

(CT) PZT for precision control with stepper motor offload (hard actuator)

(PP) PZT for precision control with hydraulic offload (hard actuator)

The words "soft" and "hard" refer to the stiffness of the actuator when the servo power is turned off. As will be seen in the analysis the soft actuator is better at reducing vibration transmission from below, and the hard actuators are better at rejecting wind disturbances from above.

The decision process for the PSA actuator selection is summarized in Figure 2. The VC actuator has been selected for further development and eventual implementation. Both the CT and PP actuators are viable fallbacks should unforeseen problems occur with the current development.

\footnotetext{
${ }^{1}$ The acronyms are TMT $=$ Thirty Meter Telescope, PSA $=$ Primary Segment Assembly, $\mathrm{VC}=$ voice coil, $\mathrm{CT}=$
} conventional technology, $\mathrm{PP}=$ piezo-pump, $\mathrm{PZT}=$ piezo-electric transducer, $\mathrm{CSI}=$ control structure interaction. 


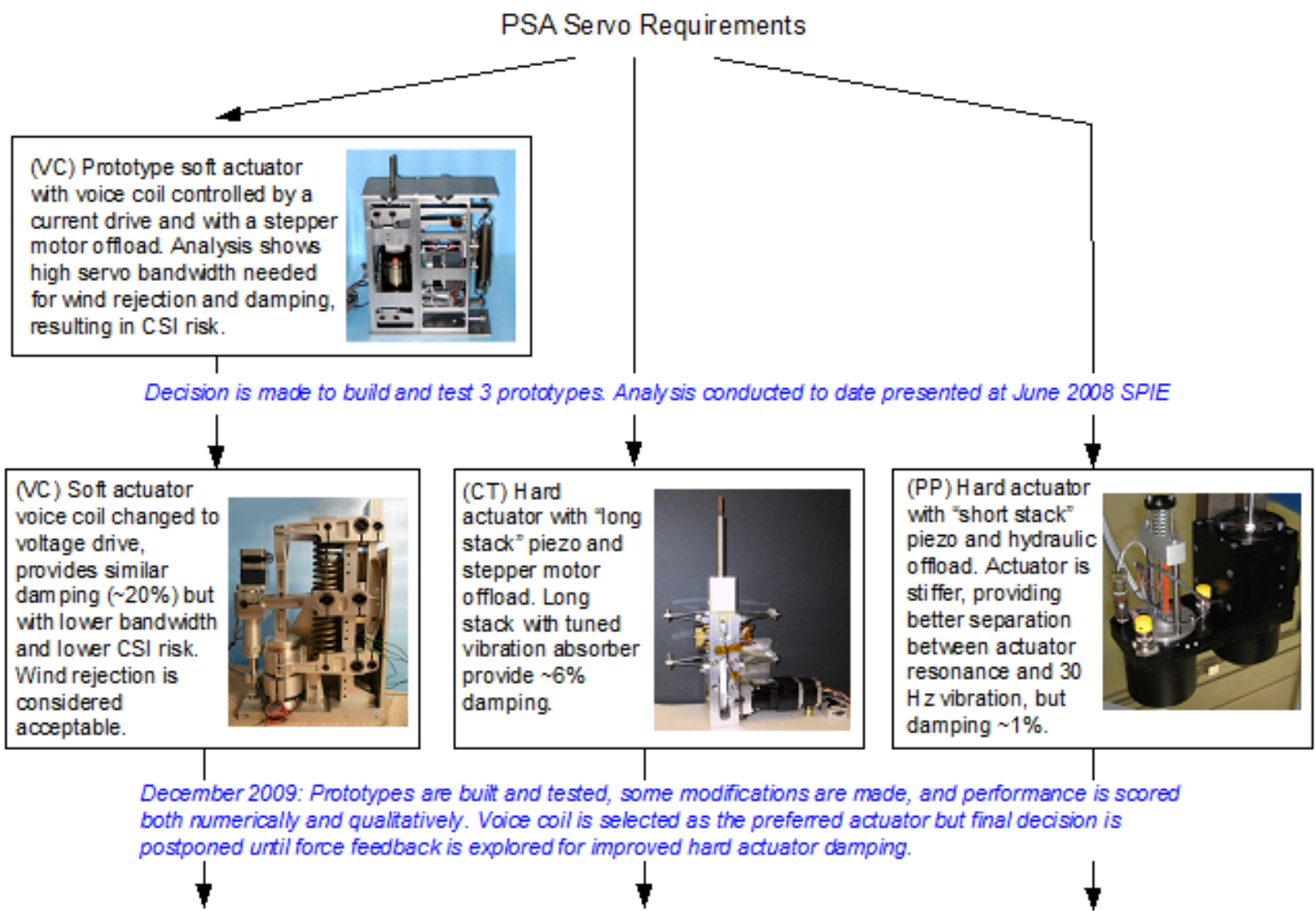

Altemative 2 phase stable servo implemented for voice coil, providing higher bandwidth with less CSI risk, better damping $(>20 \%)$ and better wind rejection.
Piezo stack for both hard actuator designs changed to two short piezo stacks, one used for actuation and the second for force sensing. High resolution control of the two hard actuators now is essentially the same and the difference between the two approaches is in the offload mechanism. Damping is improved and demonstrated to be $\sim 10 \%$. Analysis shows even higher damping is possible on a rigid base but the CSI risk becomes unacceptable.

February 2010: All three prototype actuators now considered adequate for meeting PSA servo requirements and the final decision is besed on risk assessment. Hard actuators have a higher risk of poor vibration rejection, particularly if the required actuator stiffness is not achieved and the actuator resonance lines up with or is close to the $30 \mathrm{~Hz}$ vibration due to rotating machinery. Mainly for this reason the decision is made to select the voice coil actuator. No preference is made between the different offioad approaches for the non-selected hard actuators. The voice coil is preferred with or without using the phase stable servo architecture. The main risk of the voice coil is lower bandwidth due to CSI resulting in poor wind rejection. Analysis is presented at June 1010 SPIE in this paper.

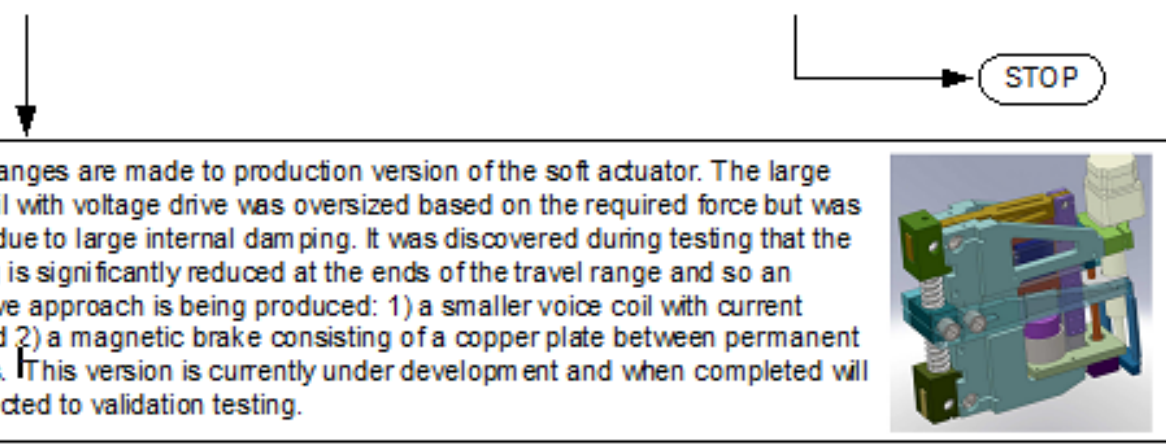

Figure 2: Decision Process for TMT PSA Actuator Down Selection 
The work reported here builds upon previous studies of the M1CS servo control loop ${ }^{[3]}$ and the global control loop. ${ }^{[4]}$ Companion papers describe wind and disturbance models, ${ }^{[5]}$ the prototype PSA built for testing and model verification, ${ }^{[6]}$, and the current status of the M1CS global loop. ${ }^{[7]}$

Section 2 describes the control design, starting with the requirements, then discusses the models, including actuator models, describes the Zernike method used for CSI analysis, and finishes by defining the compensators.

Section 3 presents the servo loop analysis. Stability of the soft actuator is assessed on both a rigid and flexible base. Comparisons are made with the hard actuator in order to provide some background for the selection process.

Section 4 summarizes the process by which the actuator decision was made.

\section{CONTROL DESIGN}

\section{Requirements}

The requirements that determine the servo control design are listed in Table 1. The requirements are challenging. The top-level requirements are stated in terms of image quality and tracking error. The image quality is measured using a statistical point spread function (PSSN), for which a perfect value is unity. The second level of requirements is more directly used for the control design. These requirements flow down from the top level and include some interpretation to translate the numbers into control system quantities such as bandwidth. The wind rejection and vibration requirements are qualitative in the sense that the objective is to maximize certain quantities. Numbers are given as goals, with the understanding that the top-level requirements are dominant. The $30 \mathrm{~Hz}$ number is important because vibration near this frequency is expected due to rotating machinery, specifically 4-pole induction motors operating at $60 \mathrm{~Hz}$. The actual frequency will be somewhat less depending on the power demand and resulting slippage, with $29.5 \mathrm{~Hz}$ being a value commonly measured during operation of the Keck telescope. Two of the requirements address the high order, multivariable nature of the control problem. The Control Structure Interaction (CSI) requirement is stated using singular values. The A-matrix is the interaction matrix between the edge sensors and the segment actuators. The $\mathrm{B}$ matrix is the pseudo-inverse of A. Errors in the A-matrix change the gain of the global loop. There are other requirements for the PSA actuators not listed in Table 1 that specify the size, weight, fit, power, cost, lifetime, and maintainability.

Table 1: PSA Actuator Requirements

\begin{tabular}{|cc|}
\hline Image quality & Tracking \\
PSSN $>0.9894$ (segment dynamic displacement & Error $<4.5 \mathrm{~nm}$ RMS \\
due to wind, vibration, actuator and sensor noise) & Range $5 \mathrm{~mm}$ \\
\hline Stability & Global loop \\
Gain margin $>10 \mathrm{~dB}$ & Bandwidth $\geq 1 \mathrm{~Hz}$ \\
Phase margin $>45 \mathrm{deg}$ & A-matrix uncertainty acceptable \\
CSI: max singular value of sensitivity $<2$ & Goal is max singular value of (I-BA) $\leq 1 / 2$ \\
Wind rejection & Vibration \\
Maximize low frequency stiffness & Maximize damping, especially near $30 \mathrm{~Hz}$ \\
Goal is $>10 \mathrm{~N} /$ micron for frequencies $<1 \mathrm{~Hz}$ & Goal is $>10 \%$ damping of principle mode \\
\hline
\end{tabular}

\section{Control System Architecture}

The signals and systems are all connected in Figure 3. At the bottom of the block diagram there is a dividing line between control and structure.

Start with the structure. Pictures of the one PSA and part of the TMT block are in Figure 1. The PSA box in the block diagram is meant to be a diagonal set of 492 segments. The TMT box is the entire telescope structure, except for the segments, including both the Az and El axes and the pier. Internal to the TMT box is the mount control system and the pier connection to the soil on Mauna Kea. The TMT finite element model is built with each PSA represented as a lumped mass connected to points on the mirror cell. The masses are mathematically removed and then the TMT and PSA blocks are connected using the stiff springs labeled $k_{b}$, which represents a set of bolts. The exogenous inputs to the structure are the force due to the wind $\left(f_{w}\right)$, the force due to the actuators $\left(f_{a}\right.$ and $\left.f_{x}\right)$, and vibration $\left(z_{v}\right)$. The vibration is 
modeled as a translation between mirror cell and the segments, an artificial "floating floor" upon which the segments sit. A more accurate model of vibration is force originating from many sources on and around the telescope. The floating floor artifice serves the purpose of modeling the vibration transmission through the PSA onto the surface of the mirror. The point of view as servo control designers is that, however the vibration gets to the base on the PSA, the actuators should seek to attenuate these vibrations.

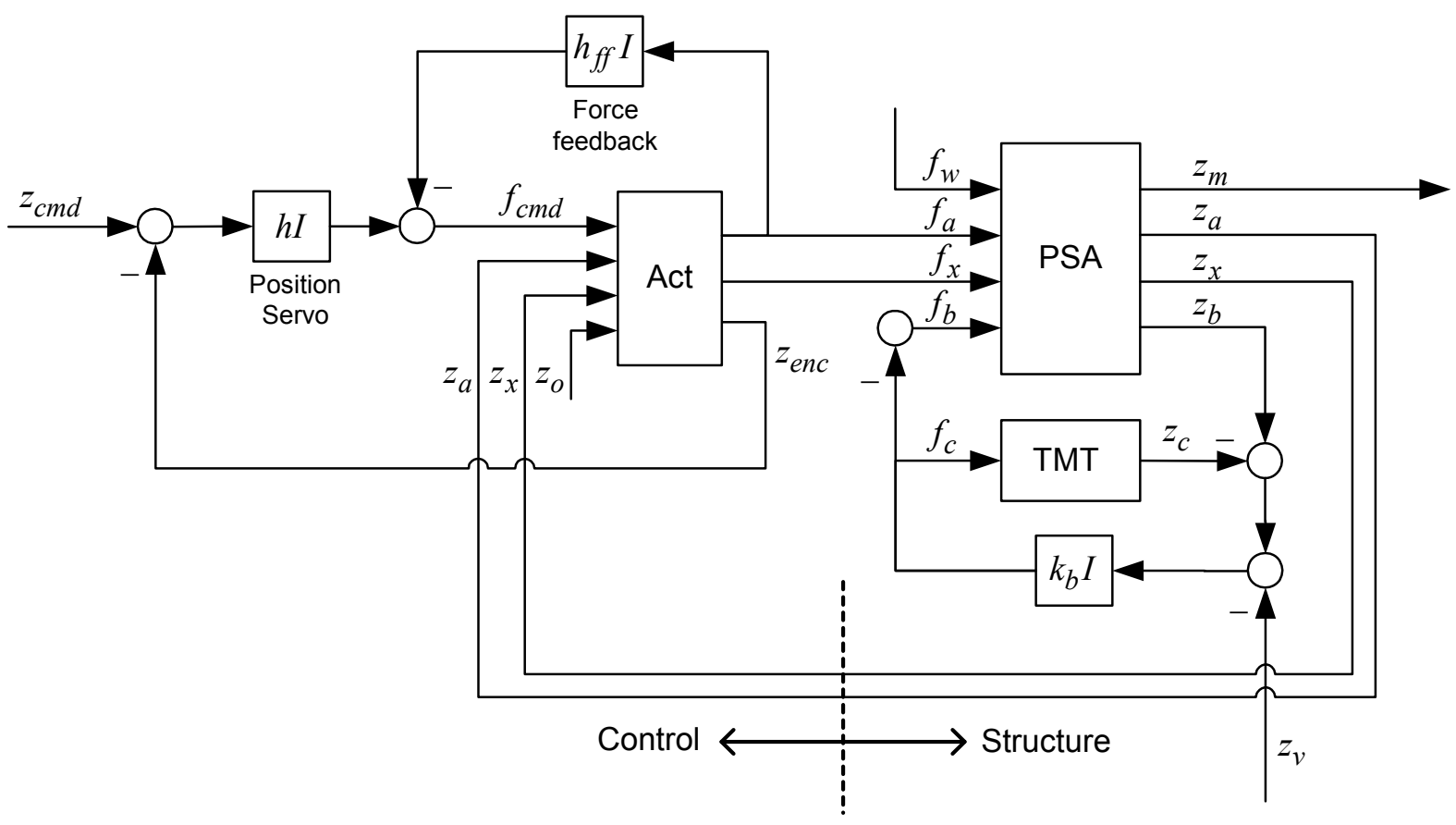

Figure 3: PSA Servo Control System Architecture

The left side of the Figure 3 block diagram is the egocentric world of the control engineer. Everything is centered on the box labeled "Act," a diagonal set of 1476 actuators. Yes there is a connection to the structure, but what is actually being controlled by the position servo is the encoder signal $z_{\text {enc }}$. The force feedback compensation is an inner loop, which makes it all a more interesting control problem.

\section{Signals and Systems}

The small letter $f$ is a vertical force, the small letter $z$ is a vertical position, and a single letter subscript refers to an absolute force or position at a node. By "absolute" it is meant that the quantity is relative to an inertial frame of reference. The signals in Figure 3 are defined below:

$f_{w}, z_{m}=$ wind force at selected nodes on the mirror cell

$f_{a}, z_{a}=$ nodes on moving frame where the output flexures

of the actuators are attached

$f_{x}, z_{x}=$ nodes on fixed frame where the actuator box is attached

$f_{b}, z_{b}=$ nodes on fixed frame where the fixed frame is attached to the mirror cell

$f_{c}, z_{c}=$ corresponding nodes on the mirror cell

$$
\begin{aligned}
& z_{v}=\text { vibration input } \\
& z_{o}=\text { offload position } \\
& z_{c m d}=\text { commanded position } \\
& z_{\text {enc }}=\text { intermediate point inside }
\end{aligned}
$$

actuator measured by the encoder

$f_{\text {cmd }}=$ force command from servos

Additional signals are defined as needed such as internal points within the actuator.

The signal dimensions depend on the context. The "complete" problem has been discussed so far and for the complete problem the signals in Figure 3 are all dimension 1476. For the "rigid base" problem the same block diagram can be 
used, except with the TMT box to zero, and the signal dimensions all reduce to 3. For the "Zernike problem" the larger signals and systems are each projected onto a single Zernike basis vector and (remarkably) the signal dimensions all reduce to 1 .

The systems and controllers are defined below:

$$
\begin{aligned}
& H(s)=\text { single PSA }(12 \times 12) \\
& G_{c}(s)=\text { mirror cell }(1476 \times 1476) \\
& A(s)=\text { single actuator }(2 \times 4) \\
& h(s)=\text { position compensator }(1 \times 1) \\
& h_{f f}(s)=\text { force feedback compensator }(1 \times 1)
\end{aligned}
$$

Use either the Kronecker product or identity matrix as appropriate to scale up the systems. For example, the block diagonal set of PSAs with 12 input and output signals for each segment has a total of $12 \times 492$ inputs and outputs.

\section{Structures}

A prototype of the PSA has been built for testing of the subsystems and for model validation. ${ }^{[2]} \mathrm{A}$ finite element model has been developed in NASTRAN, and model parameters have been adjusted to provide a better match with static load tests. The prototype PSA has an aluminum blank in place of the mirror segment and a customized mirror cell triangle for support. A dynamic characterization of the PSA prototype has been performed and then the PSA was used to test the candidate actuators. $^{[6]}$

A modified version of the PSA model is used for the work in this paper. The modifications include (i) adjustments to the structure to provide increased stiffening, (ii) changes to the attachments to match those of the mirror cell, and, (iii) most importantly, the replacement of the top part of the PSA with a mirror. The NASTRAN model is simplified using the Craig-Bampton method and then ported to Matlab for dynamic analysis. The Craig-Bampton method matches boundary conditions at selected nodes over a frequency range. Fictitious nodes are automatically inserted in order to match the boundary conditions. For this work 27 physical nodes and $350 \mathrm{~Hz}$ were selected, and the reduced order model has 261 modes.

The telescope finite element model has 1476 nodes located on the mirror cell. ${ }^{2}$ The ANSYS model is ported to Matlab using modal truncation out to the first 3000 modes.

Example frequency responses of the PSA are shown in Figure 1c, underneath a picture of the structure. The collocated PSA response is $z_{a} / f_{a}$, which is on the moving frame where the output flexure of the actuator is attached. The blue line is the response when the moving frame is supported by an offload spring that matches the one in the soft actuator, and the green line shows the effect of the soft actuator damping. The piston and tip-tilt modes respectively at $9.7 \mathrm{and} 11.0 \mathrm{~Hz}$ are damped onto the real axis, and there is also significant damping of the PSA structural modes starting at $147 \mathrm{~Hz}$.

Two versions of the mirror cell frequency response are shown in Figure 1d. The blue line is the piston response, computed with the unit-norm Zernike vector for piston, and the green line is the vertical frequency response at one randomly selected location on the mirror cell. The piston response has dominant modes at 1.90 and $4.87 \mathrm{~Hz}$ and starts to roll-off after the $4.87 \mathrm{~Hz}$ mode. The piston response can be thought of as one Newton of force spread evenly over the mirror cell (1/1476 $\mathrm{N}$ at each node) and then the output at each node is averaged. For the response at a single point, the same modes are present, but the overall response does not start to roll off until $53.2 \mathrm{~Hz}$. The interpretation is that the mirror cell is stiff at one location, but compliant overall. At a single point the response is dominated by the mirror cell, which is designed to be very stiff. The same load spread evenly around the mirror cell produces a response that is dominated by the support structure, which in absolute terms is very stiff, but relative to the single point response, is much more compliant.

\footnotetext{
${ }^{2}$ The finite element models used here are 608 model of the PSA, so-called because it was delivered to the controls team on June 8, 2009, and the 2008 DSL model of the telescope.
} 


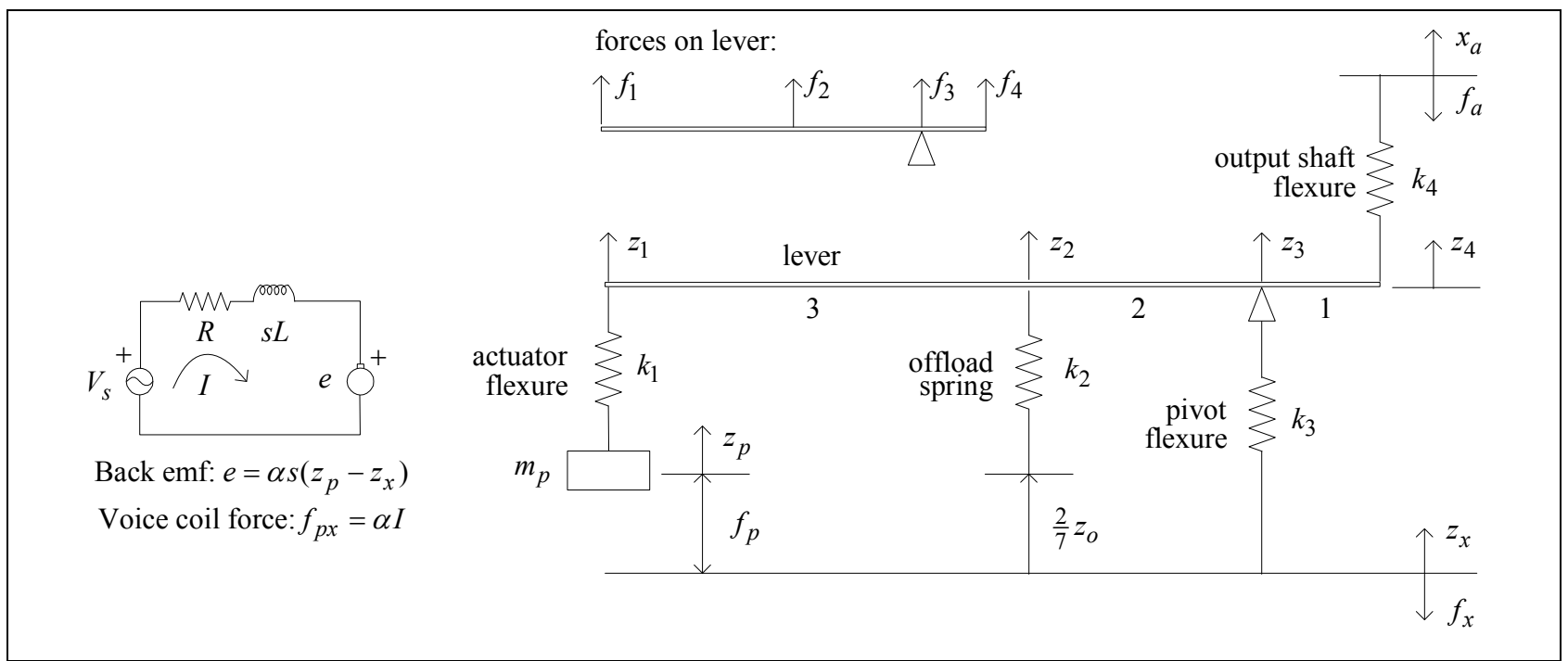

Figure 4: Diagram of Soft Actuator Voice Coil and Lever

\section{Dynamic model of Voice Coil and Lever}

A mechanical diagram is shown in Figure 4. The movable part of the voice coil has a 5:1 lever ratio and the offload spring is connected with a 2:1 lever ratio. The bottom line in the diagram is the common point where the actuator frame connects to the PSA fixed frame. The symbol $s$ is the Laplace variable. The mechanical and electrical equations from the diagram are listed below:

$$
\begin{array}{ll}
m_{p} s^{2} z_{p}=-f_{p}-f_{1} \text { (force balance on voice coil) } & f_{2}=k_{2}\left(z_{x}+(2 / 7) z_{o}-z_{2}\right) \text { (offload spring) } \\
0=f_{1}+f_{2}+f_{3}-f_{4} \text { (force balance on lever) } & f_{3}=k_{3}\left(z_{x}-z_{3}\right) \text { (pivot flexure) } \\
0=5 f_{1}+2 f_{2}+f_{4} \text { (torque balance on lever) } & f_{4}=k_{4}\left(z_{4}-z_{a}\right) \text { (output flexure) } \\
\left(z_{1}-z_{3}\right)=5\left(z_{3}-z_{4}\right) \text { (voice coil lever ratio) } & V=(R+s L) I-\alpha s\left(z_{p}-z_{x}\right) \text { (voice coil circuit) } \\
\left(z_{2}-z_{3}\right)=2\left(z_{3}-z_{4}\right) \text { (offload spring lever ratio) } & f_{p}=\alpha I \text { (voice coil force) } \\
f_{1}=k_{1}\left(z_{p}-z_{1}\right) \text { (voice coil flexure) } & f_{c m d}=(5 \alpha / R) V \text { (commanded force) }
\end{array}
$$

These are a mixed set of algebraic and differential equations, which after some algebra can be reduced to a state space model of the actuator $A(s)$. The inputs are $f_{c m d}, z_{a}, z_{x}, z_{o}$ and the outputs are $f_{a}, f_{x}, z_{\text {enc }}$.

The parameter values are measured. The flexures are $k_{1}=5 \mathrm{e} 6, k_{3}=30 \mathrm{e} 6$, and $k_{4}=25 \mathrm{e} 6 \mathrm{~N} / \mathrm{m}$. The offload spring is $k_{4}=$ $0.26 \mathrm{e} 6 / 4 \mathrm{~N} / \mathrm{m}$. The voice coil moving mass (the magnet assembly in this design) is $1.9 \mathrm{~kg}$, the force constant is $\alpha=$ $21.36 \mathrm{~N} / \mathrm{A}$. The resistance and inductance are respectively $R=1.2 \Omega$ and $L=3.8 \mathrm{mH}$. The voice coil break frequency is $R /(2 \pi L)=50 \mathrm{~Hz}$. A negative impedance op-amp circuit reduces by half the measured voice coil resistance of $2.4 \Omega$.

\section{Dynamic model of PZT Actuator}

The corresponding mechanical and electrical diagram for the hard actuator with force feedback is shown in Figure 5. Working from top to bottom: The spring is the actuator output flexure, the top PZT is used as a force sensor, the bottom PZT is a force producer, and the bottom spring is the stiffness of the actuator body. The governing equations are:

$$
\begin{array}{ll}
f_{a}=f_{1} \text { (force on moving frame) } & \left.f_{1}=f_{2} \quad \text { (force balance at } z_{1}\right) \\
\left.f_{1}=k_{1}\left(z_{e}-z_{a}\right) \text { (force balance at } z_{e}\right) & I_{2}=s C V_{2}-s d f_{2} \quad \text { (actuation PZT electrical) } \\
I_{1}=s C V_{1}-s d f_{1} \text { (sensor PZT electrical equation) } & f_{2}=d k V_{2}-k\left(z_{1}-z_{2}\right) \text { (actuation PZT mechanical) } \\
f_{1}=d k V_{1}-k\left(z_{e}-z_{1}\right) \text { (sensor PZT mechanical) } & V_{s}=R_{2} I_{2}+V_{2} \quad \text { (actuation PZT circuit equation) } \\
0=R_{1} I_{1}+V_{1} \text { (sensor PZT circuit equation) } & \left.f_{2}=k_{2}\left(z_{x}+z_{o}-z_{2}\right) \text { (force balance at } z_{2}\right)
\end{array}
$$


These equations can be reduced to a state space model of the actuator. For the subsequent discussion consider the equations for a single PZT, which can be either the forcer or the sensor: ${ }^{[8],[9]}$

$$
\begin{aligned}
& I=C s V-d s f \\
& z=d V-k^{-1} f \\
& V_{s}=R I+V
\end{aligned}
$$

$$
\begin{aligned}
& I[\mathrm{Amps}]=\text { current } \\
& z[\mathrm{~m}]=\text { width } \\
& V=\text { voltage across the plates } \\
& f[\mathrm{~N}]=\text { force on the PZT }
\end{aligned}
$$

$C[$ farads $]=$ capacitance

$k[\mathrm{~N} / \mathrm{m}]=$ spring constant

$d[\mathrm{~m} / \mathrm{V}]=$ electrical-mechanical constant

$k_{33}=d \times \operatorname{sqrt}(k / \mathrm{C})$ [unitless] $=$ quality factor

The three equations are respectively the electrical, mechanical, and circuit equations. The non-dimensional term $k_{33}$ is commonly used to characterize the material, with close to one being good.

If the response is fast enough and the PZT material is stiff enough then the coupled equations reduce to $z=d V_{s}$, which is pure position control. This is the approximate model used in earlier work. ${ }^{[3]}$

If the response is fast enough but the material stiffness needs to be included, then the electrical equation can be neglected and the bottom two reduce to $f=d k V_{s}+k z$. This model suffices for the PZT forcer for all but the CT actuator.

The resistor in the PZT circuit removes energy and hence results in damping. The original CT actuator takes advantage of PZT damping. Rearrange the three coupled equations to the following:

$$
\begin{aligned}
& f=h_{1}(s) V_{s}-h_{o}(s) z, \text { where: } \\
& h_{o}(s)=\frac{-k(1+s R C)}{(1+s R C \beta)}, h_{1}(s)=\frac{d k}{(1+s R C \beta)} \\
& \beta=1-\frac{d^{2} k}{C}=1-k_{33}^{2}
\end{aligned}
$$

The transfer function $h_{\mathrm{o}}(s)$ is a lead compensator, and it is the resulting phase lead that creates damping. The amount depends on both the location and the separation of the pole and zero. The location is set by the resistor value. The basic idea is the dominant pole of the system should be in the middle of the lead compensator pole and zero. The separation depends on the $k_{33}$ constant, which is a property of the material, and as the value of $k_{33}$ becomes closer to 1 there is more separation and hence more damping. The only way to change the $k_{33}$ value is to change the type of PZT material.

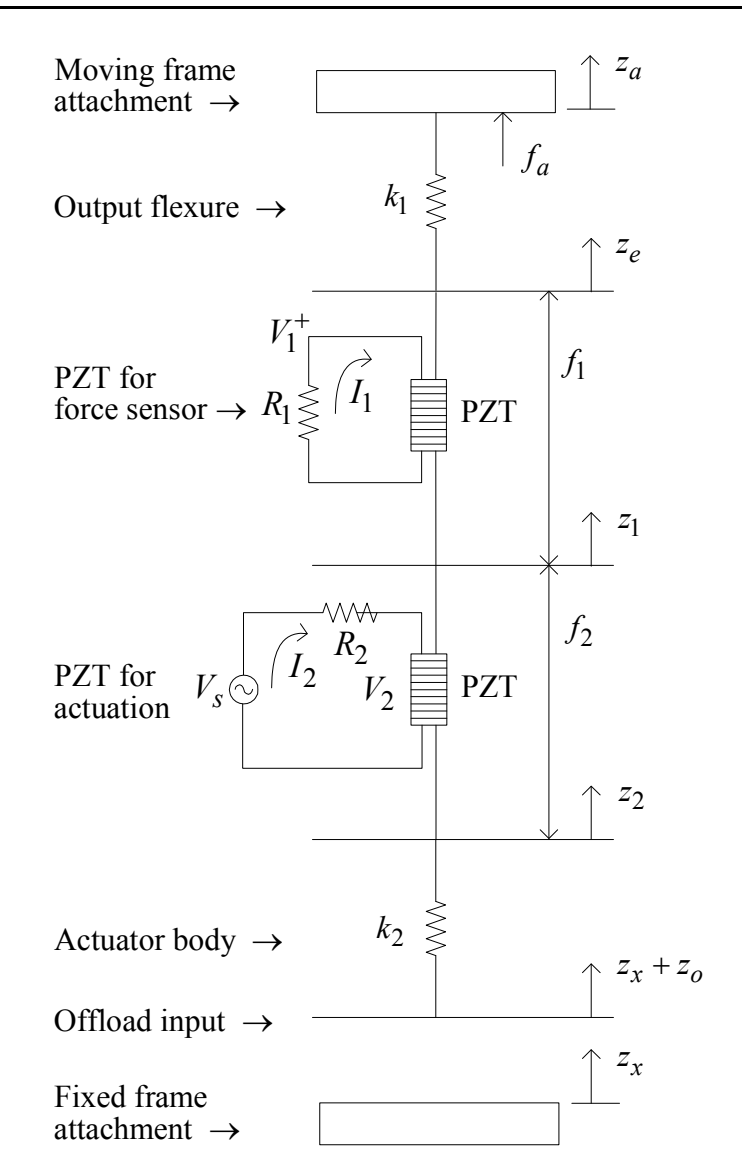

Figure 5: Diagram of Hard Actuator

The resistor $R$ can be replaced by an op-amp circuit. In this case replace $R$ in the above equations with a complex impedance $Z$. The op-amp circuit can be designed to provide more damping over a narrow frequency range.

In practice a significant amount of damping can be achieved by an isolated PZT, but it is the damping of the PZT in combination with the rest of the system that matters. To achieve good overall damping the PZT must be the most compliant element in the system. Define the ratio $k_{s} / k$ as the stiffness of the system divided by the PZT stiffness. The "ratio-rule" is $\zeta_{s} \approx\left(k_{s} / k\right) \zeta$, in other words the damping of the system is reduced by the ratio of the stiffness (the actual relationship is more complicated, but the ratio-rule provides good insight). The practical effect is that a large amount of local damping is needed to overcome the ratio-rule, and this requires a large amount of PZT material (in order to be adequately compliant yet accommodate the loads). This is the approach used by the CT actuator, which uses a "long stack" of PZT material. Further discussion of the amount of damping that was achieved for the CT actuator is presented in Section 4. 
A PZT can also be used as a force sensor. Connect the plates with a resistor and measure the voltage across the resistor. Solve the coupled equations and the result is $V=s R d /(1+s R C) f$, called a "washout." At high frequencies above $1 /(R C)$ the measured voltage is proportional to force, and at low frequencies the voltage is proportional to the derivative of force. In the time domain, the voltage measurement due to a constant force washes out to zero. This turns out to be a good thing, and if the washout did not happen naturally it would need to be designed into the force feedback compensator, otherwise the wind rejection is compromised. In the control design the resistance value is selected in order to put the break frequency $1 /(R C)$ at a good location.

The PZT equations and the force balance equations from Figure 5 can be combined into a transfer function or state space model of the actuator. This level of complexity is needed to properly model the original CT compensator, but a simpler version suffices for the force feedback design. The drive PZT is assumed fast enough so that its electrical equation can be ignored, and the force PZT is replaced with an exact force measurement, with the understanding the implementation will include the washout term. For the force feedback design the actuator model reduces to the following:

$$
f_{a}=f_{c m d}-k_{o}\left(z_{a}-z_{x}-z_{o}\right),
$$

where $k_{o}$ is the series combination of the springs, and the commanded force is proportional to the PZT voltage. This is the version of the hard actuator used in the analysis section. The model descriptions are complete, and attention turns to the problem of control structure interaction (CSI).

\section{Zernike Method for CSI Design and Analysis}

The mirror cell is a flexible base and care must be taken in the servo design to not induce oscillations in the mirror cell. The design and analysis of the CSI problem is complicated by the high order of the combine PSA and TMT systems. A simplifying approach is used here called the "Zernike Method." It is discussed and used in our earlier work, ${ }^{14}$ and here a different interpretation and brief summary is given. Consider the block diagram in Figure 6 with the $B$ and $B^{T}$ operators applied respectively to the inputs and output.

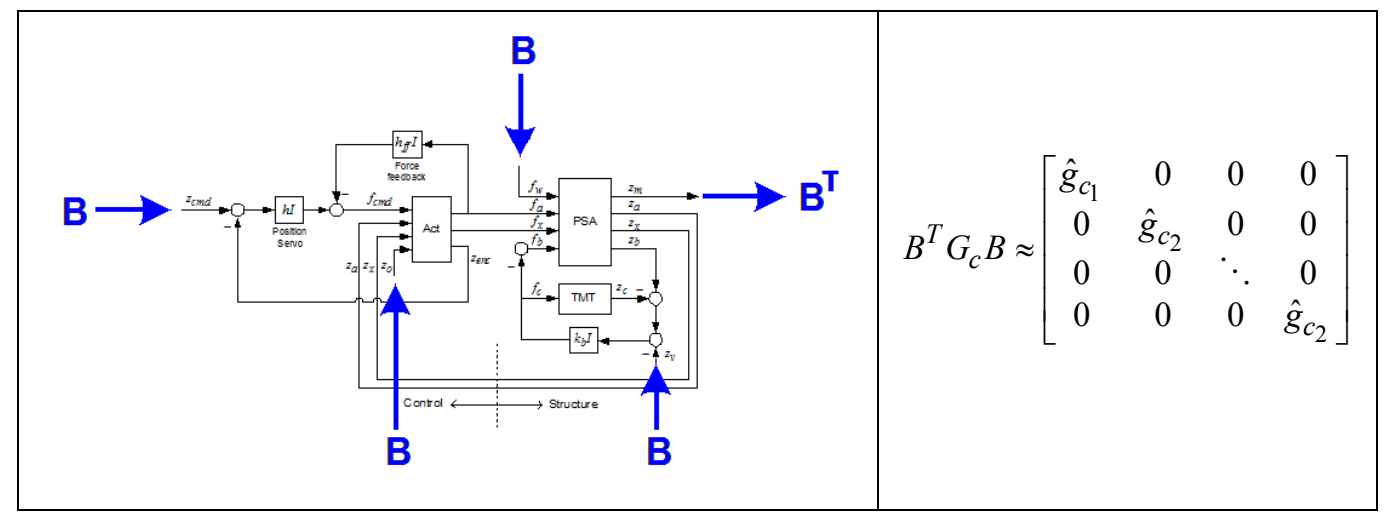

Figure 6: Transformation used for Zernike Method

The $B$ matrix contains the Zernike basis vectors, piston, tip, tilt, and so on for a total of 1476 basis vectors. Perform block diagram algebra, or the corresponding matrix algebra, and then approximate the mirror cell system as a diagonal set of single input single output (SISO) systems. Each of these SISO systems is the mirror cell model projected onto a Zernike basis vector. Our earlier work has shown this is a good approximation. Similarly assume that the collection of PSA models become diagonal. This is again not exact, but a good approximation, especially for the low order Zernike vectors. The actuator and controller blocks exactly diagonalize. The result of these transformations is that one large problem $(1476 \times 1476)$ is replaced by 1476 SISO problems. The block diagram in Figure 6 (the same as in Figure 3) can be used to represent any one of the SISO problems.

The Zernike method is (1) Compute the Zernike projections and (2) Perform the control design and analysis on each of the transformed systems. The key observation is that the same controller must work for all of the systems. The control design is an iterative process until the stability and robustness requirements are satisfied for all of the Zernike systems.

Our experience has been, more often than not, that a control design that passes all of the requirements on a rigid base will show sensitivity to one or more of the Zernike systems. No sensitivity has been found beyond radial degree 10 , and 
so out of the 1476 possible Zernike systems, analyzing at most 50 suffices. The calculations are fast, less than one second for each Zernike system.

The drawback of the Zernike method is that stability of the SISO systems does not guarantee stability of the multivariable system. For this reason the control system design must be checked using singular value methods. This calculation is addressed in the earlier and current companion papers. ${ }^{[4],[7]}$

\section{The Control Design}

Classical methods are used for the control design. For a given compensator various frequency responses are computed and then adjustments are made to the compensator to change the shape of the frequency response. This method is called "loop shaping." For the soft actuator the force feedback is not used and the position compensator is:

$$
h(s)=\frac{17.47 s^{3}+6289 s^{2}+5.644 \mathrm{e} 005 s+2.2 \mathrm{e} 007}{2.068 \mathrm{e}-007 s^{3}+0.0009095 s^{2}+\mathrm{s}}=\frac{8.451 \mathrm{e}+007[0.7671,11.26](40)}{(0)(350)^{2}}[\mathrm{~N} / \mathrm{m}]
$$

For the hard actuator the actuator stiffness used is $k_{o}=17 \mathrm{~N} /$ micron. The position and force feedback compensators are:

$$
\begin{aligned}
& h(s)=\frac{2.013 \mathrm{e} 010}{s^{2}+62.83 s}=\frac{2.013 \mathrm{e}+010}{(0)(10)}[\mathrm{N} / \mathrm{m}] \\
& h_{f f}(s)=\frac{0.1201 s^{2}+251.3 s}{2.533 \mathrm{e}-007 s^{4}+0.001783 s^{3}+1.221 s^{2}+132.6 s+3948}=\frac{4.742 \mathrm{e}+005(0)(333)}{(10)^{2}(100)(1000)} \text { [unitless] }
\end{aligned}
$$

The transfer functions are given both in polynomial and shorthand form. ${ }^{3}$ The shorthand form gives pole and zero frequencies in $\mathrm{Hz}$ and the damping ratios of the complex modes. For example: (350) is a real mode at $350 \mathrm{~Hz}$, and $[0.7671,11.26]$ is a complex mode at $11.26 \mathrm{~Hz}$ with $76.71 \%$ damping.

\section{CONTROL ANALYSIS}

\section{Loop Transfer Function}

The loop transfer function (LTF) is the gain around the loop defined at the position compensator. Two versions of the LTF for the soft actuator are shown in Figure 7. Part (a) is for a rigid base, defined at one of the three compensators with the other two loops open. The unit magnitude crossover is $9 \mathrm{~Hz}$ and the robustness margins are all well above the requirements. The high gain at low frequency is made possible by allowing the LTF response near $80 \mathrm{~Hz}$ to be near unity. This is not a stability problem because the sensor and actuator are collocated and the phase rises in this frequency range. This type of design is called a "phase stable" design. Part (b) shows the corresponding LTFs using the Zernike method. The Zernike systems are listed in the plot. The frequency range around $9 \mathrm{~Hz}$ is labeled as a risk because the robustness of one of the Zernike systems has decreased (the tip mode). The risk is that a further decrease in robustness may set up a tip oscillation in the mirror cell. The frequency range around $80 \mathrm{~Hz}$ is labeled as a risk because the gain has increased above unity.

\section{Wind Response}

The wind responses for the soft and hard actuators are compared in Part (c). This is a rigid base response with all three actuators closed. The plot is the $z_{m} / f_{w}$ piston response. The objective is low gain below $1 \mathrm{~Hz}$. For the hard actuator the low frequency gain is determined mainly by the actuator stiffness. The low frequency gain for the soft actuator is determined mainly by the compensator integral gain. The soft actuator does not quite meet the goal of $10 \mathrm{~N} / \mathrm{micron}$ at 1 $\mathrm{Hz}$, coming in at about $7 \mathrm{~N} /$ micron, which is considered acceptable. Clearly the hard actuator has an advantage.

\footnotetext{
${ }^{3}$ The shorthand form is defined by $a(s+2 \pi b)\left[s^{2}+2 \zeta \omega s+\omega^{2}\right]=a(b)[\zeta, f]$, where $f=\omega /(2 \pi)$.
} 


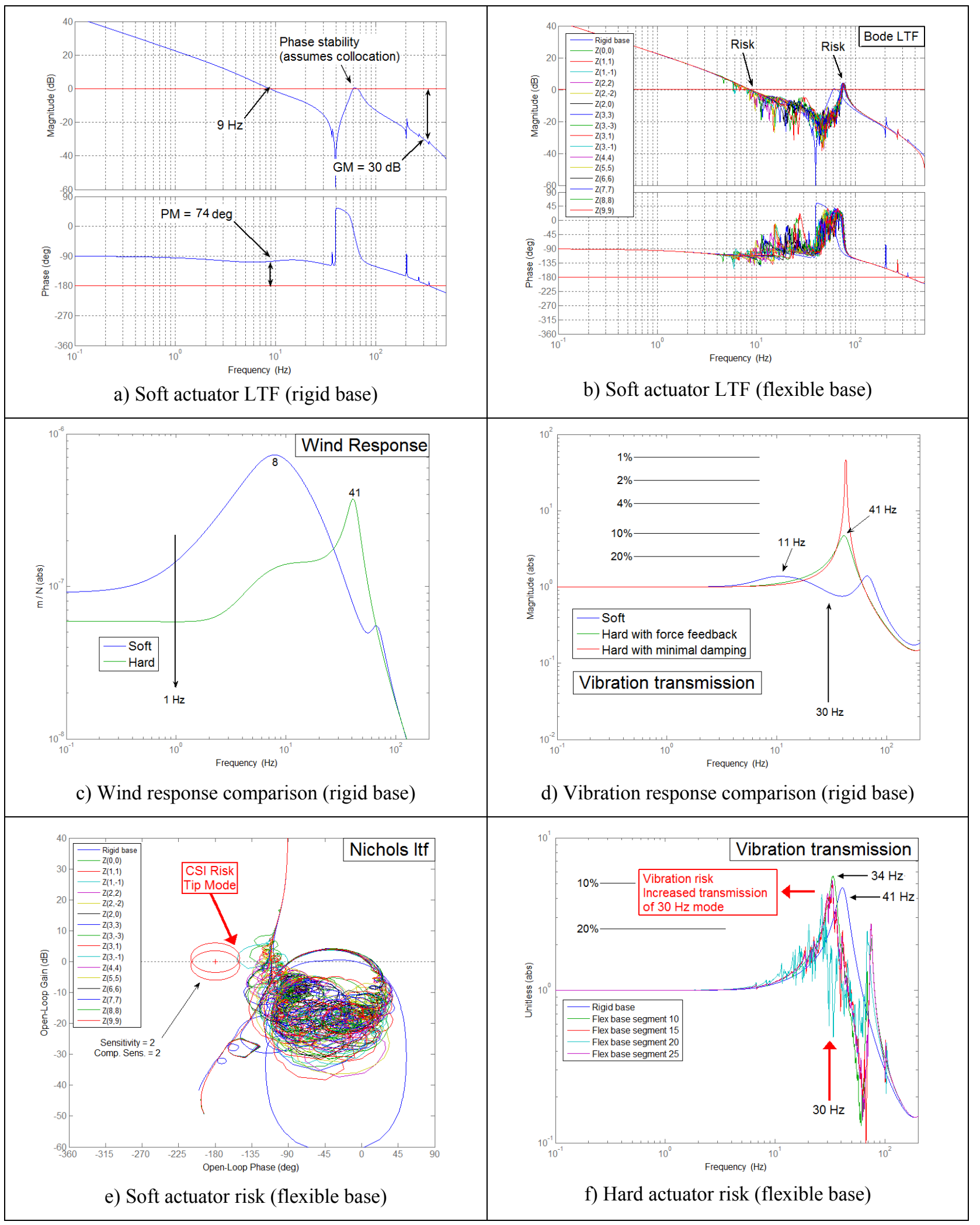

Figure 7: Control System Analysis

Proc. of SPIE Vol. 7733 77332F-11 


\section{Vibration Response}

The vibration responses for the soft and hard actuators are compared in Part (d). This is again a rigid base response with all three actuators closed. The plot is the $z_{m} / z_{v}$ piston response. At low frequencies the response is very near unity. Vibrations at low frequency pass straight through the PSA and the base and mirror move together. At high frequencies the vibration response is attenuated. Base vibrations in this range result in much smaller vibrations at the mirror. The important part of the vibration response is the amplification that occurs around the dominant mode, which is $11 \mathrm{~Hz}$ for the soft and $41 \mathrm{~Hz}$ for the hard actuator. The vertical scale is the amplification scaled using the damping ratio where the amplification $A=1 /(2 \zeta)$. High gain is not necessarily a problem if there is no vibration energy at that frequency. Clearly the soft actuator has an advantage.

\section{Risks}

Risks of the soft and hard actuators are shown respectively in Parts (e) and (f).

The soft actuator plot in Part (e) is the same Zernike LTF responses in Part (b) but plotted on a Nichols chart. The Nichols chart is gain versus phase and gives a good visual representation of robustness. The overlapping circles in the middle are sensitivity and complementary sensitive equal to 2 . The objective is to avoid these circles, and it is seen that one of the Zernike responses crosses over. It is not a stability issue, because the lines do not go past the cross in the middle of the plot. This is a CSI risk. Higher gain causes a tip oscillation in the main mirror cell. Lower gain reduces the risk, at the cost of less wind rejection.

The hard actuator plot in Part (f) is the vibration response on a flexible base. The responses are the vibration transmission at different mirror cell locations. The dominant frequency drops from $41 \mathrm{~Hz}$ to $34 \mathrm{~Hz}$, and sometimes less. The comfortable margin above $30 \mathrm{~Hz}$ has become much smaller. This drop is due to the compliance of the mirror cell, but the same would happen if an actuator for whatever reason becomes more compliant. The risk is that the dominant structural mode and the dominant vibration might line up. Choosing the soft actuator eliminates this risk.

\section{ACTUATOR SELECTION}

\section{Damping of the Hard Actuators}

In the course of studying and testing the prototype actuators several different approaches for improved hard actuator damping were tried.

The original PP actuator is a PZT actuator with a "short stack" that is very stiff and very fast. A consequence is that the PZT does not have a large range of travel, and so periodic offloads are needed. This was not a problem because the hydraulic design proved to be both fast and smooth, satisfying the tracking requirements. There was however no separate mechanism in the original design for damping. A small amount is provided by the mechanical and hydraulic components, measured in the $1-2 \%$ range. This is the basis for the tall red response in Figure $7 \mathrm{~d}$.

The original CT actuator used a "long stack" PZT actuator, which made it possible to use the PZT for passive damping. The damping is available over a narrow range of frequency and the resistor value was adjusted to match the dominant response of the closed loop PSA system. In the CT tests the shunt resistor provided about $4 \%$ damping to the overall system. This was less than expected, and the reason was that the effective material constant $k_{33}$ of the packaged actuators incorporated into the mechanical amplifier was less than the raw material constant.

Development continued by replacing the resistor with a tuned op-amp circuit, and in this way the measured damping was increased to about $6 \%$. The drawback of a tuned circuit is the frequency range where maximum damping is achieved becomes smaller, and so narrow that adaptive tuning might be required.

At some point in the process a reference was located that reported on similar PZT damping experiments. ${ }^{[9]}$ Alas, all of our approaches have been tried before, including force feedback, plus one more, an op-amp circuit providing negative capacitance. That too was tried, but was no better than the tuned circuit.

It was at this point that the controls team started to explore force feedback. The "original" designs for both hard actuators were replaced by "updated" designs using force feedback. We did not want to give up on the hard actuator, because the hard actuator has a clear advantage for wind rejection. Alan Schier of The Pilot Group, the developer of the PP actuator, proposed the use of force feedback to the controls group, which spurred the development. The force feedback has two advantages. The $k_{33}$ parameter that defines the PZT material is no longer a limit to the damping that can be achieved, and 
the deleterious ratio-rule no longer appliers. The updated PP and CT actuators with force feedback have essentially the same PZT implementation for precision control, with the same amount of damping, and differ only in the offload mechanism. Both became viable candidates. The measured damping was increased to $10 \%$. More damping is available for a single PSA on a rigid base, but the value that was used for the actuator comparison was limited to $10 \%$ due to concerns about CSI robustness.

\section{The Selection Process}

A decision process was developed that was partly numerical, partly pass/fail, and partly qualitative. The process is summarized in Figure 8. The numerical scores for nominal and worst case performance are based on image quality (PSSN values). A numerical score for reliability and risk was also used.

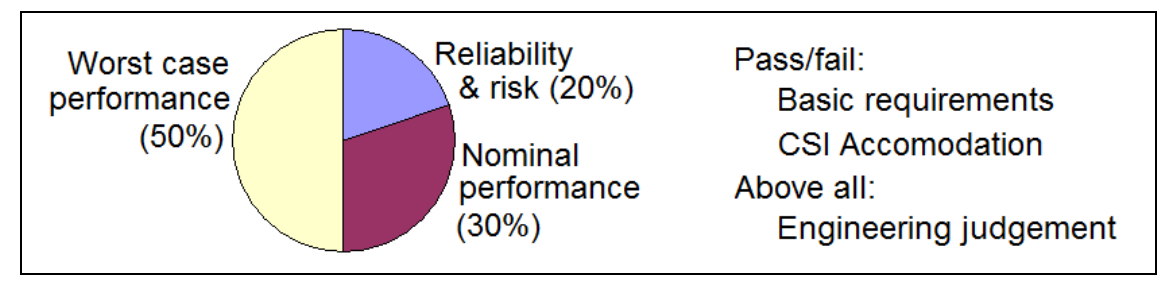

Figure 8: The Process for Actuator Scoring

All three of the candidate actuators were found to meet the pass/fail requirements, to have good nominal performance, and to have acceptable reliability and risk. All three were considered viable candidates. This put the controls team and the whole project in a good position. The deciding factor was the worst-case performance. The worst-case soft actuator (poor wind performance) was rated higher than the worst-case hard actuator (poor vibration performance). The decision was made to proceed with the soft actuator. Both of the hard actuators are good alternatives should unforeseen development problems occur with the soft actuator.

\section{CONCLUSIONS}

The PSA actuators are one of the critical components in the TMT telescope. A large design effort for the actuators is summarized in this paper. Three candidate actuators were built, tested, improved, and tested again.

Testing has been used to validate many of the requirements. These include the tracking requirements, stability on a rigid base, wind rejection, and vibration transmission. Designing and completing these tests has been a major achievement.

Several of the requirements cannot be tested today and must wait until the TMT structure is built. These requirements include CSI robustness, global loop bandwidth, and most importantly, image quality. The un-testable requirements are analyzed using models. The middle part of this paper describes several of the models and the control system analysis based on these models. Contributions of this paper include the models for the soft and hard actuators.

The soft actuator has been selected for further development and that process is now under way.

\section{ACKNOWLEDGEMENTS}

The TMT Project gratefully acknowledges the contribution of the following actuator manufactures: The Pilot Group for the Piezo Pump Actuator (inquiries@the-pilot-group.com), Marjan Research (mvjean@marjanresearch.com) for the voice-coil soft actuators, and the Jet Propulsion Laboratory (www.jpl.nasa.gov) for the CT actuator.

The work described in this paper was carried out in part at the Jet Propulsion Laboratory, California Institute of Technology, under contract with the National Aeronautics and Space Administration. The authors wish to thank the Thirty Meter Telescope Observatory Corporation for funding this work.

The TMT Project gratefully acknowledges the support of the TMT partner institutions. They are the Association of Canadian Universities for Research in Astronomy (ACURA), the California Institute of Technology and the University of California. This work was supported as well by the Gordon and Betty Moore Foundation, the Canada Foundation for Innovation, the Ontario Ministry of Research and Innovation, the National Research Council of Canada, the Natural Sciences and Engineering Research Council of Canada, the British Columbia Knowledge Development Fund, the Association of Universities for Research in Astronomy (AURA) and the U.S. National Science Foundation. 


\section{REFERENCES}

[1] Nelson, J. and Sanders, G. H., "The Status of the Thirty Meter Telescope Project," Proc. SPIE 7012, 70121A (2008).

[2] Williams, E. C., Baffes, C., Mast, T., Nelson, J., Platt, B., Ponchione, R. J. Ponslet, E., Setoodeh, S., Sirota, M., Stephens, V., Stepp, L., Tubb, A., "Advancement of the Segment Support System for the Thirty Meter Telescope Primary Mirror,” Proc. SPIE 7018-37, (2008).

[3] Thompson, P. M., MacMynowski, D. G., and Sirota, M. J., "Control Analysis of the TMT Primary Segment Assembly,” Proc. SPIE 7012-58 (2008).

[4] MacMynowski, D. G., Thompson, P. M., and Sirota, M. J., “Analysis of TMT Primary Mirror Control Structure Interaction,” Proc. SPIE 7017-41, (2008).

[5] MacMynowski, D. G., Colavita, M. M., Skidmore, W., and K. Vogiatzis, "Primary mirror dynamic disturbance models for TMT: Vibration and Wind," Proc. SPIE 7738-14, (2010).

[6] Regehr, M. W., Thompson, P. M., Colavita, M. M., Moore, J. D., Sirota, M., and Williams, E. C., "Dynamic Characterization of a prototype of the Thirty Meter Telescope primary segment assembly," Proc. SPIE 7733-85, (2010).

[7] MacMynowski, D. G., Thompson, P. M., Shelton, C., and Roberts, Jr. L. C., "Robustness of Thirty Meter Telescope Primary Mirror Control,” Proc. SPIE 7733-189, (2010).

[8] Hagood, N. W. and A. von Flotow, "Damping of Structural Vibrations with Piezoelectric Materials and Passive Electrical Networks," Journal of Sound and Vibration 146(2), 243-268 (1991).

[9] Preumont, A., B. de Marneff, A. Deraemaeker, and F. Bossens, "The damping of a truss structure with a piezoelectric transducer," Computers and Structures, 86, 227-239, www.sciencedirect.com (2008). 